University of Nebraska - Lincoln

DigitalCommons@University of Nebraska - Lincoln

Virology Papers

Virology, Nebraska Center for

2012

\title{
Chloroviruses: Not Your Everyday Plant Virus
}

James L. Van Etten

University of Nebraska-Lincoln, jvanetten1@unl.edu

David D. Dunigan

University of Nebraska-Lincoln, ddunigan2@unl.edu

Follow this and additional works at: https://digitalcommons.unl.edu/virologypub

Part of the Biological Phenomena, Cell Phenomena, and Immunity Commons, Cell and Developmental Biology Commons, Genetics and Genomics Commons, Infectious Disease Commons, Medical Immunology Commons, Medical Pathology Commons, and the Virology Commons

Van Etten, James L. and Dunigan, David D., "Chloroviruses: Not Your Everyday Plant Virus" (2012). Virology Papers. 244.

https://digitalcommons.unl.edu/virologypub/244

This Article is brought to you for free and open access by the Virology, Nebraska Center for at DigitalCommons@University of Nebraska - Lincoln. It has been accepted for inclusion in Virology Papers by an authorized administrator of DigitalCommons@University of Nebraska - Lincoln. 


\title{
Chloroviruses: Not Your Everyday Plant Virus
}

\author{
James L. Van Etten and David D. Dunigan
}

Department of Plant Pathology and Nebraska Center for Virology, University of Nebraska, Lincoln, Nebraska, U.S.A.

Corresponding author - J.L.Van Etten, jvanetten1@unl.edu

\begin{abstract}
Viruses infecting higher plants are among the smallest viruses known and typically have four to ten protein-encoding genes. By contrast, many viruses that infect algae (classified in the virus family Phycodnaviridae) are among the largest viruses found to date and have up to 600 protein-encoding genes. This brief review focuses on one group of plaque-forming phycodnaviruses that infect unicellular chlorella-like green algae. The prototype chlorovirus PBCV-1 has more than 400 protein-encoding genes and 11 tRNA genes. About $40 \%$ of the PBCV-1 encoded proteins resemble proteins of known function including many that are completely unexpected for a virus. In many respects, chlorovirus infection resembles bacterial infection by tailed bacteriophages.
\end{abstract}

\begin{abstract}
Algal viruses
Viruses infecting higher plants are typically small RNA viruses that encode only a few genes [1]. Although small viruses have recently been discovered that infect algae (e.g. [2]), many viruses infecting eukaryotic algae are huge dsDNA viruses with genomes ranging from 160 to $560 \mathrm{~kb}$ with up to 600 protein-encoding genes (CDSs) and are the subject of this review. These large viruses (family Phycodnaviridae), are found in aqueous environments throughout the world and play dynamic, albeit largely undocumented, roles in regulating algal communities such as the termination of massive algal blooms commonly referred to as red and brown tides (e.g. [3-5]). This review focuses on one genus in the Phycodnaviridae, the chloroviruses, which are large, icosahedral, plaque-forming (Figure 1a), dsDNA-containing viruses that replicate in certain unicellular, chlorella-like green algae [6-9]. As noted below, their structure, their initial stages of infection, and many of their genes resemble bacteriophages more than viruses that infect eukaryotes, i.e. they are not your everyday plant virus.
\end{abstract}

\section{Chloroviruses}

Chloroviruses exist in freshwater throughout the world with titers as high as 100,000 plaque-forming units (PFU) per $\mathrm{ml}$ of indigenous water, although titers are typically 1-100 PFU/ml. Titers fluctuate during the year with the highest titers occurring in the spring [7,9]. Chlorovirus hosts, which are normally symbionts and are often referred to as zoochlorellae, are associated with the protozoan Paramecium bursaria (Figure 1b), the coelenterate $\mathrm{Hy}$ dra viridis or the heliozoon Acanthocystis turfacea [8].
Zoochlorellae are resistant to viruses in their symbiotic state. Fortunately, some zoochlorellae grow independently of their partners, permitting plaque assay of the viruses and synchronous infection of their hosts, which allows one to study the virus life cycle in detail. Three such zoochlorellae are Chlorella NC64A (recently renamed Chlorella variabilis [10], and its viruses are called NC64A viruses), Chlorella SAG 3.83 (renamed Chlorella heliozoae, and its viruses are called SAG viruses), and Chlorella Pbi (renamed Micratinium conductrix, and its viruses are called Pbi viruses). However, little is known about the natural history of the chloroviruses and we suspect that many more chlorovirus hosts and viruses exist in nature.

Paramecium bursaria chlorella virus (PBCV-1), which infects C. variabilis (Figure $1 \mathrm{c}, \mathrm{d}$ ), is the type member of the genus Chlorovirus [7]. The C. variabilis $46.2 \mathrm{Mb}$ genome was sequenced recently [11] and the availability of both host and virus sequences makes chloroviruses an attractive model system. Given the coding capacity of the chloroviruses, it is not surprising that they encode many unusual proteins. However, with the exception of homologs solely in other chlorovirus members, the majority of their CDSs do not match anything in the databases. Some of these viruses also have introns and inteins, which are rare in viruses.

\section{PBCV-1 structure}

Cryo-electron microscopy and fivefold symmetry averaging three-dimensional reconstruction of PBCV-1 indicate the outer capsid is an icosahedron and covers a single lipid bilayered membrane, which is required for infection (Figure 1e and f) [12-14]. One of the PBCV-1 vertices has a $560 \AA$ long spike-structure; $340 \AA$ protrudes from the surface of the virus. The part of the spike structure that is outside the capsid has an external diameter of $35 \AA$ at the tip, expanding to $70 \AA$ at the base (Figure $1 \mathrm{~g}$ and $\mathrm{h}$ ). The spike structure widens to 160 $\AA$ inside the capsid and forms a closed cavity inside a large pocket between the capsid and the membrane enclosing the virus DNA (Figure $1 \mathrm{~h}$ ). Therefore, the internal virus membrane departs from icosahedral symmetry adjacent to the unique vertex (Figure 1f). Consequently the virus DNA located inside the envelope is packaged non-uniformly in the particle.

The capsid shell consists of 1680 donut-shaped trimeric capsomers plus 11 pentameric capsomers, one at each icosahedral vertex except for the spike-containing vertex. The trimeric capsomers are arranged into 20 tri- 

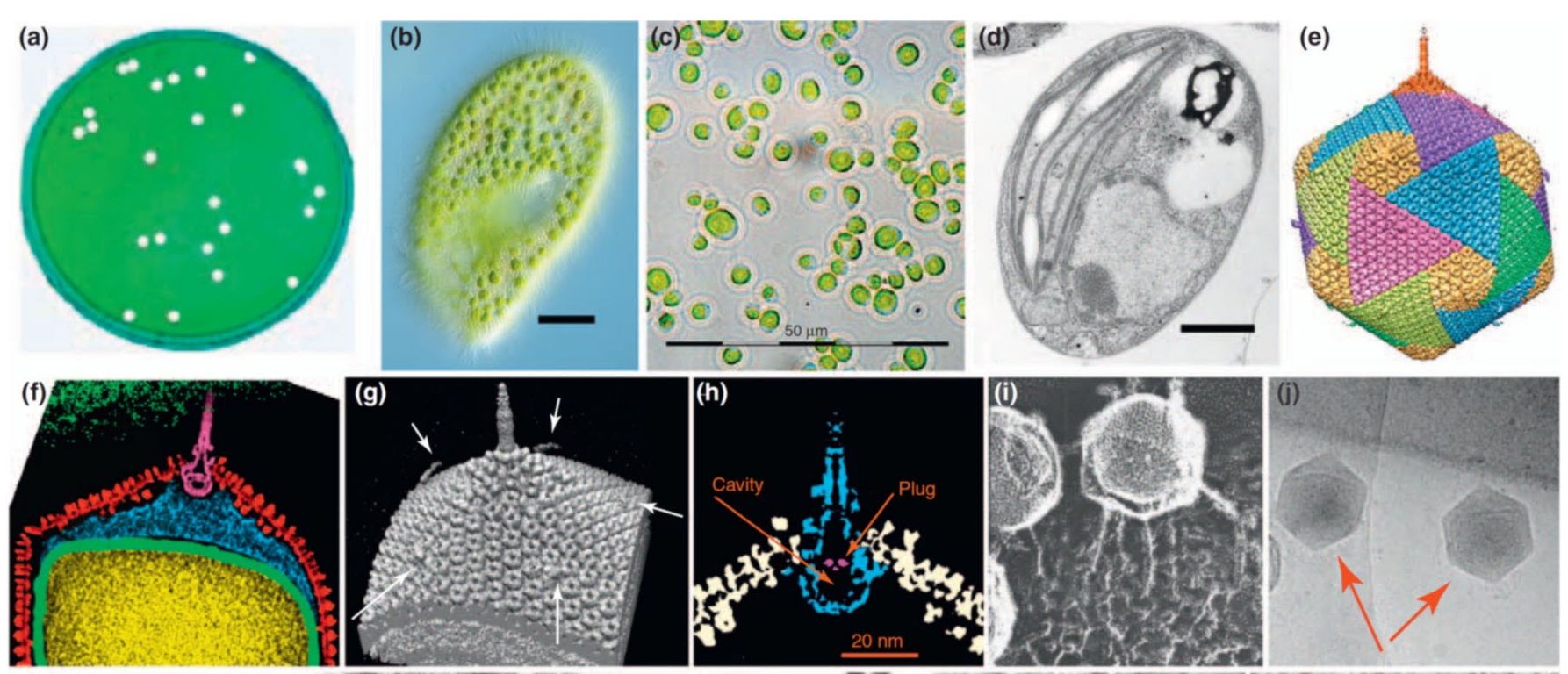

(k)
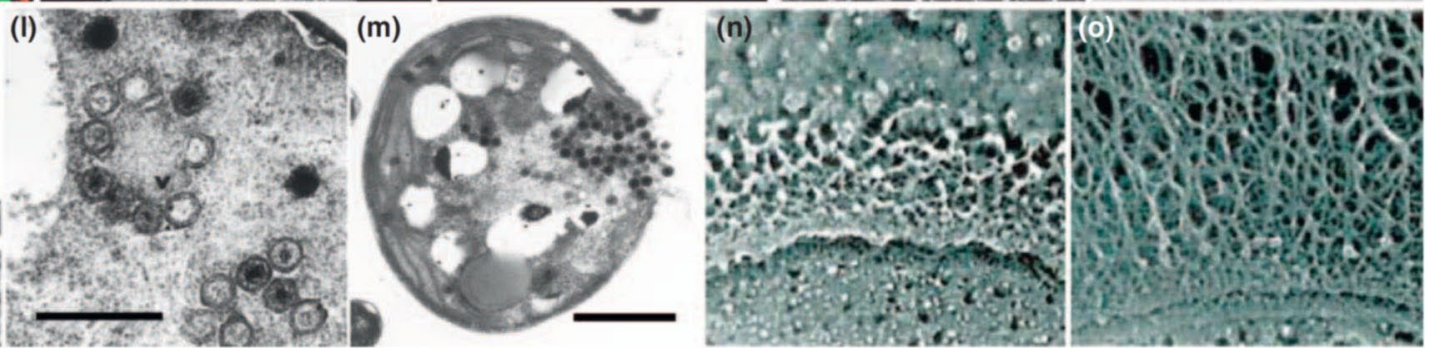

Figure 1. Chlorella cells and chlorovirus PBCV-1. (a) Plaques formed by PBCV-1 on a lawn of $C$. variabilis. (b) Paramecium bursaria and its symbiotic chlorella cells (size bar: $20 \mathrm{~mm}$ ). (c) Chlorella variabilis cells cultured in the laboratory free of both Paramecium and virus (size bar: $50 \mathrm{~mm}$ ). (d) Thin section of a healthy C. variabilis cell (size bar: $1 \mathrm{~mm}$ ). (e) Fivefold averaged cryo-electron micrograph of PBCV-1 reveals a long narrow cylindrical spike structure at one vertex (orange) and fibers extending from one unique capsomer per trisymmetron. The virion has a diameter varying from $1650 \AA$, measured along the twofold and threefold axes, and $1900 \AA \AA$, measured along the fivefold axes. The glycoprotein shell is composed of 20 triangular units, or 'trisymmetrons,' and 12 pentagonal caps, or 'pentasymmetrons,' at the fivefold vertices (yellow). (f) Central cross-section of (e). Note the gap between the unique vertex and the membrane enclosing the DNA. (g) Surface view of the PBCV-1 spike structure and fibers. (h) A magnified image of the spike structure (in blue). Also note a plug structure (in pink) inside the spike (size bar: $20 \mathrm{~nm}$ ). (i) PBCV-1 attached to the cell wall as viewed by the quick-freeze, deep-etch procedure. Note the virions attached to the wall by fibers. (j) Initial attachment of PBCV-1 to C. variabilis cell walls. The orientation of the unique vertex (recognized by the pocket under the vertex) can be seen for the two virus particles marked with red arrows. These virus particles have their unique vertex facing the cell wall indicating that these particles recognize the cell surface. (k) Attachment of PBCV-1 to the algal wall and digestion of the wall at the point of attachment. This occurs within 1-3 min p.i. (size bar: $100 \mathrm{~nm}$ ) (I) Virion particles assemble in defined areas in the cytoplasm named virus assembly centers. Note both DNA containing (dark centers) and empty capsids (size bar: $500 \mathrm{~nm}$ ). ( $\mathrm{m}$ ) Localized lysis of cell plasma membrane and cell wall and release of progeny viruses at $\sim 8 \mathrm{~h}$ p.i. (size bar: $1 \mathrm{~mm}$ ). (n/o) Chlorella variabilis cell wall surface of uninfected ( $\mathbf{n}$ ) and $4 \mathrm{~h}$ after PBCV-1 infection (o), as viewed by quick-freeze, deep-etch electron microscopy. Note the accumulation of a dense, fibrous hyaluronan network on the surface of the infected cells. Figure b was kindly provided by Wim van Egmond; Figure e from [13]; Figure $\mathrm{f}$ was kindly provided by Xinzheng Zhang and Michael Rossmann; Figures $\mathrm{g}$, $\mathrm{h}$ and $\mathrm{j}$ from [14]; Figure i from [26]; Figure $\mathrm{k}$ from [83], Figures I and $\mathrm{m}$ from [84], and Figures $n$ and o from [56] - all published with permission.

angular facets (trisymmetrons, each containing 66 trimers) and 11 pentagonal facets (pentasymmetrons, each containing 30 trimers and one pentamer at the icosahedral vertices) (Figure 1e). External fibers extend from some of the trisymmetron capsomers (probably one per trisymmetron) and presumably aid in virus attachment to the host (Figure 1i).

The PBCV-1 major capsid protein (MCP) is a glycoprotein and consists of two eight-stranded, antiparallel $\beta$-barrel jelly-roll domains. The monomeric MCP forms a trimeric capsomer, which has pseudo-sixfold symmetry [15]. Identities of the other virus surface proteins are unknown. A proteomic analysis of highly purified PBCV-1 virions indicates that the particle has 148 virusencoded proteins (Dunigan et al., unpublished).

\section{PBCV-1 life cycle}

Three-dimensional reconstruction of PBCV-1 in the presence of $C$. variabilis cell walls established that the spike first contacts the cell wall (Figure 1j) and that the fibers then aid in holding the virus to the wall (Figure 1i) [14]. PBCV-1 attachment to its host receptor is specific and attachment is a major factor in limiting its host range. The spike is too narrow to deliver DNA and likely serves to puncture the wall and is then jettisoned. Following host cell wall degradation by a virus-associated enzyme(s) (Figure 1k), the viral internal membrane presumably fuses with the host membrane, facilitating entry of the viral DNA and virion-associated proteins into the cell, leaving an empty capsid attached to the surface [16]. This fusion process triggers rapid depolarization of the host membrane, possibly by a virus-encoded $\mathrm{K}^{+}$channel (named $\mathrm{Kcv}$ for $\underline{\mathrm{K}}^{+}$channel from chlorella virus) predicted to be in the internal membrane of the virus releasing $\mathrm{K}^{+}$from the cell. The rapid loss of $\mathrm{K}^{+}$ and associated water fluxes from the host reduce its turgor pressure, which may aid ejection of viral DNA and virion-associated proteins into the host [16]. Host mem- 


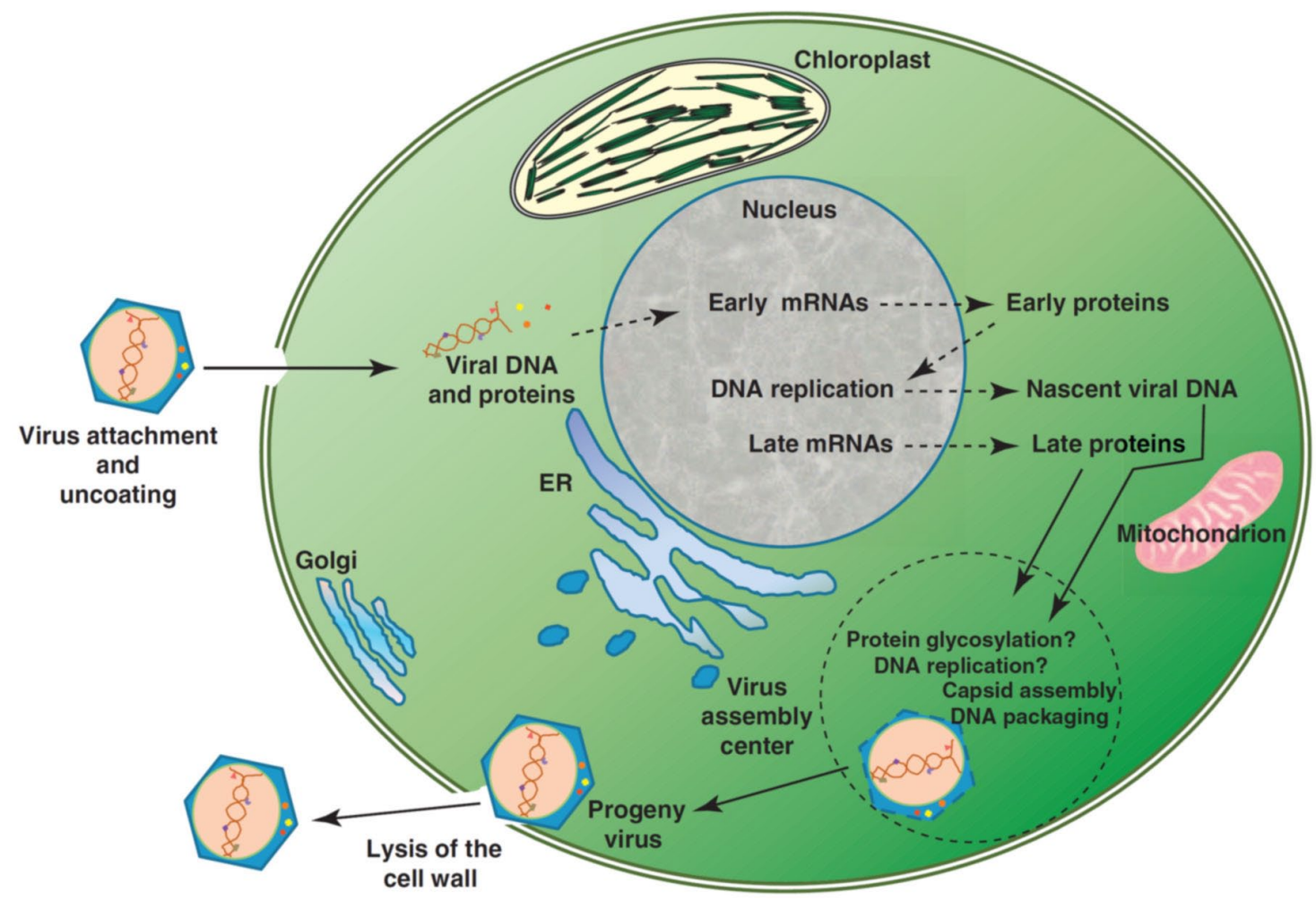

Figure 2. Proposed replication cycle of PBCV-1. The virus uncoats at the surface of the alga and the viral DNA, possibly with associated proteins, is assumed to move to the nucleus where early gene transcription begins within 5-10 min p.i. The early mRNAs are transported to the cytoplasm for translation, and at least some early proteins presumably return to the nucleus to initiate viral DNA replication, which begins 60-90 min p.i., followed by late gene transcription. Late mRNAs are transported to the cytoplasm for translation and many of these late proteins are targeted to the virus assembly centers, where virus capsids are formed and DNA is packaged. The chlorella cell membrane and wall lyses, and infectious PBCV-1 progeny viruses are released at $6-8 \mathrm{~h}$ p.i. $(\rightarrow)$ Known events; $(-->)$ hypothesized events.

brane depolarization also rapidly inhibits many host secondary transporters [17] and probably prevents infection by a second virus [18].

PBCV-1 lacks a recognizable RNA polymerase gene, and so circumstantial evidence suggests PBCV-1 DNA and viral-associated proteins quickly move to the nucleus where early transcription begins $5-10$ min postinfection (p.i.) $[7,9]$.The rapid initiation of virus transcription suggests that some component(s) facilitates transport of virus DNA to the nucleus. This component could be a product of the PBCV-1 a443r gene. A443R is packaged in the virion and may be a SUMOylated protein; the predicted A443R structure resembles mammalian SUMOylated proteins involved in nuclear trafficking (Van Etten et al., unpublished).

In this immediate-early phase of infection, host transcription rates decrease [19] and the host transcription machinery is reprogrammed to transcribe viral DNA. Details of reprogramming are unknown but host chromatin remodeling is probably involved. PBCV-1 encodes a SET domain containing protein (referred to as vSET) that methylates Lys-27 in histone 3. vSET is packaged in the PBCV-1 virion, and circumstantial evidence indicates vSET represses host transcription following PBCV-1 infection [20]. In addition, host chromosomal DNA degradation begins within minutes after infection, presumably by PBCV-1 encoded and packaged DNA restriction endonuclease(s) [19]. This degradation also inhibits host transcription. The net result is that by $20 \mathrm{~min}$ p.i., $33-50 \%$ of the polyadenylated mRNAs in the in- fected cell are of viral origin (Van Etten, unpublished). This rapid increase in viral mRNAs probably involves the selective degradation of host mRNAs, by an unknown mechanism(s), as well as viral transcription.

Viral DNA replication begins $60-90 \mathrm{~min}$ p.i. and is followed by transcription of late genes [7]. About 2-3h p.i., assembly of virus capsids begins in localized regions in the cytoplasm, which become prominent 3-4 h p.i. (Figure 11). DNA packaging of empty virus particles may involve a virus-encoded DNA packaging ATPase [21]. However, the putative DNA packaging ATPase is part of a larger CDS (A392R), which consists of two predicted fused proteins separated by a caspase cleavage site. We hypothesize that virus DNA packaging begins after a host/virusencoded enzyme with caspase-like activity cleaves A392R to produce a functional enzyme (Van Etten et al., unpublished). Note: Earlier studies on the phycodnavirus Emiliana huxleyi virus established that a caspase-like cleavage activity of one or more virus-encoded proteins was required for its replication [22,23].At 5-6 h after PBCV-1 infection the cytoplasm fills with infectious progeny virus particles and localized lysis of the host cell releases progeny at $6-8 \mathrm{~h}$ p.i. (Figure 1m). Each infected cell releases $\sim 1000$ particles, of which $\sim 30 \%$ form plaques. A schematic diagram of the PBCV-1 replication cycle is reported in Figure 2.

Global transcription of PBCV-1 genes during virus replication was examined by microarrays [24] and the results can be summarized as follows: (i) $98 \%$ of the PBCV-1 protein-encoding genes are expressed in labo- 


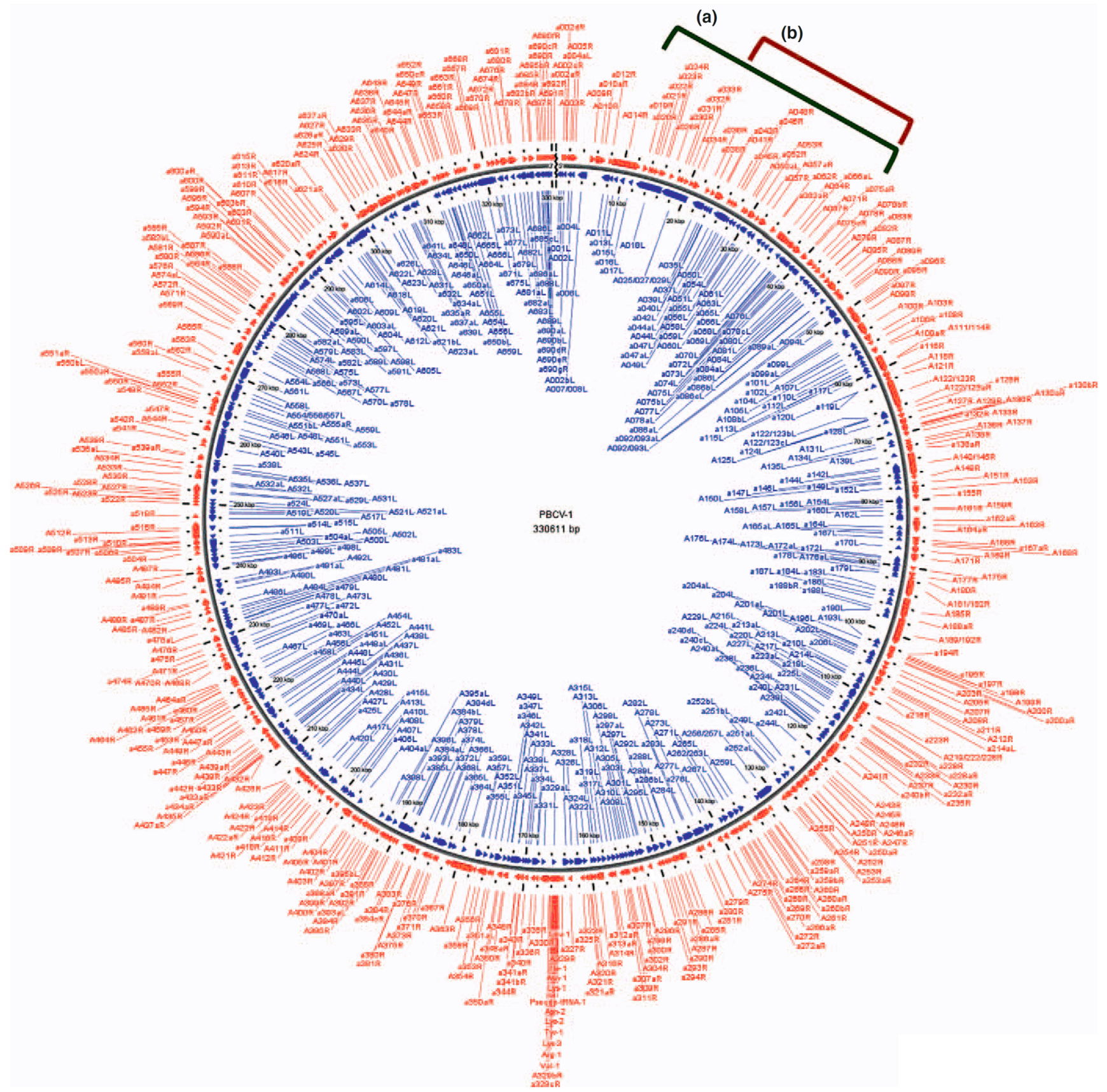

Figure 3. Map of the resequenced and annotated PBCV-1 genome (GenBank accession number JF411744.1) presented as a circle. However the genome is a linear molecule and the ends are depicted at the top ( 12 o'clock) of the figure as a crooked black line. Sequences to the right of the black line are numbered from 1 to 330611 base pairs. Open reading frames (ORF) are indicated by the block arrows. Those pointing clockwise (red) are annotated with an 'R,' those pointing counter-clockwise (blue) are annotated with an 'L.' The gene tags are annotated with the prefix ' $a$ ' indicating the ORF is unlikely to be expressed, or with ' $A$ ' indicating that it is likely to be expressed, and in most cases shown to be expressed at the RNA level and in many cases as proteins. The polycistronic tRNA gene is located near ' 6 o'clock.' The two brackets on the top-right of the figure depict two classes of deletion mutants; (a) deletion of map position 4.9-42.2, (b) deletion of map position 16-42.2. The figure was composed using CGView [85].

ratory conditions; (ii) $63 \%$ of the genes are expressed before $60 \mathrm{~min}$ p.i. (early genes); (iii) $37 \%$ of the genes are expressed after $60 \mathrm{~min}$ p.i. (late genes); and (iv) $43 \%$ of the early gene transcripts are also detected at late times following infection (early/late genes). Many of the late and early/late genes encode virion-associated proteins, consistent with particle assembly and maturation.

\section{Chlorovirus genomes}

The PBCV-1 genome is a linear $\sim 331-\mathrm{kb}$, nonpermuted dsDNA molecule with covalently closed hairpin ter-
mini.Identical $\sim 2.2-\mathrm{kb}$ inverted repeats flank each hairpin end. The remainder of the PBCV- 1 genome contains primarily single-copy DNA [7]. The GC content of the PBCV-1 genome is $\sim 40 \%$; by contrast, its host nuclear genome is $\sim 67 \%$ GC. The PBCV-1 genome was recently re-sequenced to correct mistakes in the original sequence. Using 40 codons as the minimum CDS size, PBCV-1 contains 416 predicted CDSs (Figure 3) (Dunigan et al., unpublished; GenBank JF411744). Of the predicted CDSs, $\sim 40 \%$ resemble proteins of known function, including many that are novel for a virus. The 
CDSs are evenly distributed on both DNA strands with minimal intergenic spaces. One exception is a 1788-nucleotide sequence in the middle of the PBCV-1 genome encoding 11 tRNAs, which is cotranscribed as a large precursor and then processed to mature tRNAs [9].

Not all PBCV-1 genes are required for virus replication. Four spontaneously derived PBCV-1 mutants were isolated that contain 27-to 37-kb deletions at the left end of the genome [25]. Taken together, $\sim 40 \mathrm{~kb}$ of singlecopy DNA encoding 31 CDSs, or $12 \%$ of the PBCV-1 genome, is not essential for PBCV-1 replication in the laboratory, although its replication is attenuated. Deleted CDSs include two putative capsid-like proteins, a putative glycerophosphoryl diesterase, a putative D-lactate dehydrogenase, a glycosyltransferase and a pyrimidine dimer-specific glycosylase.

The chlorella virus genomes contain methylated bases. Genomes from 37 chloroviruses have 5-methylcytosine $(5 \mathrm{mC})$ in amounts ranging from $0.12 \%$ to $47.5 \%$ of the total cytosines. In addition, 24 of the 37 viral DNAs contain $\mathrm{N}^{6}$-methyladenine $(6 \mathrm{~mA})$ in amounts ranging from $1.5 \%$ to $37 \%$ of the total adenines [26]. The methylated bases occur in specific DNA sequences, which led to the discovery that chloroviruses encode multiple $5 \mathrm{mC}$ and $6 \mathrm{~mA}$ DNA methyltransferases. About $25 \%$ of the virus-encoded DNA methyltransferases have companion DNA site-specific (restriction) endonucleases, including some with unique cleavage specificities that are sold commercially (e.g. [26-28]. The purpose of the other virus-encoded DNA methyltransferases is unknown.

Five additional chloroviruses have been sequenced and annotated including two viruses (NY-2A and AR158) that infect the same host as PBCV-1, C. variabilis [29], two viruses (MT325 and FR483) that infect M. conductrix [30], and one virus (ATCV-1) that infects C. heliozoae [31]. About $80 \%$ of the genes are common to all six viruses, suggesting they are important for virus replication. However, the sum total of chlorella virus encoded genes is much larger than those present in any one virus. Not surprisingly, homologs from viruses infecting the same host are the most similar; the average amino acid identity between homologs from PBCV-1 and NY2A or AR158 is $\sim 73 \%$. PBCV-1 and MT325 or FR483 orthologs have $\sim 50 \%$ amino acid identity and PBCV-1 and ATCV-1 orthologs have $\sim 49 \%$ amino acid identity. Using PBCV-1 as a model, there is good synteny among the three viruses that infect $C$. variabilis. By contrast, PBCV-1 has only slight synteny with the two Pbi viruses and the SAG virus [32]

\section{Chlorovirus genes}

Many PBCV-1 encoded enzymes are either the smallest or among the smallest proteins in their family including the smallest eukaryotic ATP-dependent DNA ligase [33], the smallest type II DNA topoisomerase [34], the smallest prolyl-4-hydroxylase [35], the smallest histone methyltransferase [36] and the smallest protein to form a functional K+ channel named Kcv [37]. Whereas K+ channels from prokaryotes and eukaryotes often consist of several hundred amino acids, Kcv from PBCV-1 consists of 94 amino acids [37] and virus ATCV-1 Kcv consists of 82 amino acids [38]. In spite of their small sizes, both viral proteins form functional channels that have many of the properties associated with larger $\mathrm{K}+$ channels such as selectivity, gating and sensitivity to inhibitors [39-42]. Kcv proteins readily assemble into tetramers and sort in cells to distinct target membranes [43]. This means that all of the structural requirements for correct assembly of the channel as well as for the basic functional properties of a $\mathrm{K}+$ channel exist in the viral Kcvs [44].

Phylogenetic analyses indicate that some of the viral minimalist proteins are evolutionary precursors of more complex cellular proteins. Despite their small sizes, the virus proteins typically have all of the catalytic properties of larger enzymes. Their small size and the fact that they are often 'laboratory friendly' have made them excellent models for mechanistic and structural studies, e.g. see $[41,42]$.

Chloroviruses encode many unexpected proteins a few examples are listed in Table 1. Some PBCV-1-encoded proteins assemble into metabolic pathways, e.g. hyaluronan synthesis, fucose synthesis and polyamine synthesis. Space only allows us to comment on a few unusual proteins. For example, in addition to the aforementioned $\mathrm{K}^{+}$channel $\mathrm{Kcv}$, the viruses encode other channel/transporter proteins including an aquaglyceroporin [45], a $\mathrm{Ca}^{2+}$-transporting ATPase [46] and a HAK/ KUP/KT-like $\mathrm{K}^{+}$transporter [47].

Presumably, polyamines play an essential role in the PBCV-1 life cycle and need to be tightly regulated because PBCV-1 encodes four functional polyamine biosynthetic enzymes, arginine/ornithine decarboxylase, agmatine iminohydrolase, N-carbamoylputrescine amidohydrolase, and homospermidine synthase [48-50]. In addition, PBCV-1 encodes a functional polyamine acetyltransferase, which is presumably involved in polyamine degradation (Van Etten et al., unpublished). These five polyamine metabolic enzymes are highly conserved among all of the chloroviruses; however, their functions are unknown.

Chloroviruses are also unusual because they often encode enzymes involved in sugar metabolism [51]. For example, two PBCV-1 encoded enzymes synthesize either GDP-L-fucose or GDP-D-rhamnose from GDP-Dmannose $[52,53]$. Other chloroviruses encode additional sugar metabolizing enzymes including a UDP-glucose-4,6-dehydratase [54] and a putative mannose6-phosphate isomerase. Three PBCV-1 encoded enzymes are involved in the synthesis of the extracellular matrix polysaccharide hyaluronan, including hyaluronan synthase (HAS) $[55,56]$. Hyaluronan, a ubiquitous constituent of the extracellular matrix in vertebrates, consists of $\sim 20,000$ alternating $\beta-1,4$-glucuronic acid and $\beta-1,3-\mathrm{N}-$ acetylglucosamine residues [57]. Until the has gene was discovered in PBCV-1, hyaluronan was thought to occur only in vertebrates and a few pathogenic bacteria $[57,58]$. PBCV-1 also encodes two enzymes involved in the biosynthesis of hyaluronan precursors, glutamine:fructose6-phosphate amidotransferase and UDP-glucose dehydrogenase [59]. All three genes are expressed early during PBCV-1 infection. These results led to the discovery that hyaluronan lyase-sensitive hair-like fibers accumulate on the surface of PBCV-1 infected host cells beginning at 15 min p.i. By $4 \mathrm{~h}$ p.i., the infected cells are 
Table 1. Selected unusual gene products encoded by the chlorella viruses

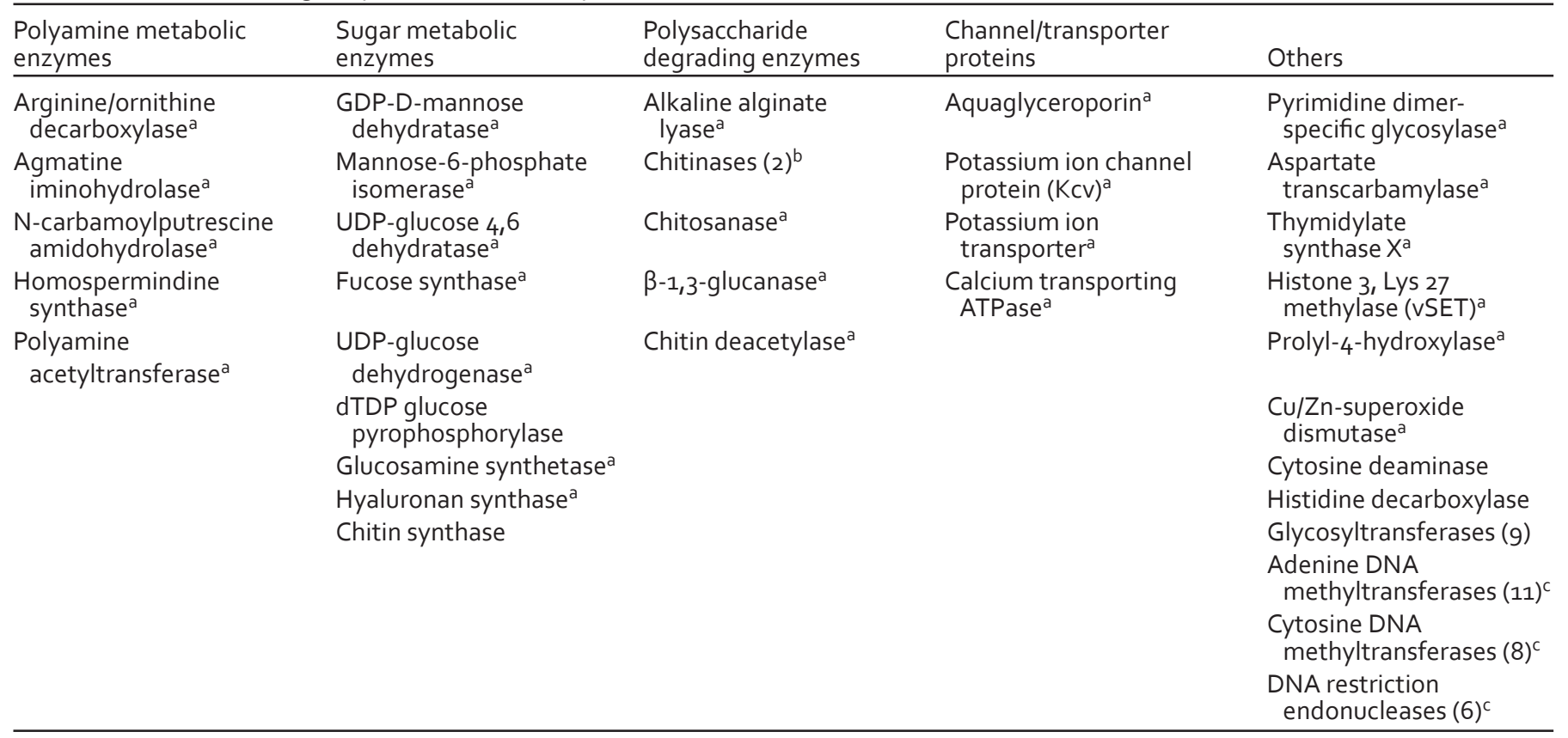

${ }^{a}$ Recombinant protein is functional.

${ }^{b}$ The number in parentheses refers to the number of different enzymes with that activity.

'Some of the recombinant proteins are known to be functional.

covered with a dense fibrous hyaluronan network (compare Figure 1n and o) [56].

The has gene is present in many, but not all, chloroviruses [56]. Surprisingly, many chloroviruses lacking a has gene have a gene encoding a functional chitin synthase (CHS). Furthermore, cells infected with these viruses produce chitin fibers on their external surface [60]. Chitin, an insoluble linear homopolymer of $\beta$-1,4-linked $\mathrm{N}$-acetyl-glucosamine residues, is a common component of insect exoskeletons, shells of crustaceans and fungal cell walls.

A few chloroviruses encode both has and chs genes and form both hyaluronan and chitin on the surface of their infected cells $[60,61]$. Finally, some chloroviruses probably lack both genes because cells infected with these viruses produce no extracellular polysaccharides [56]. The fact that many chloroviruses encode enzymes involved in extracellular polysaccharide biosynthesis suggests that the polysaccharides, which require a huge amount of ATP for their synthesis, are important in the viral life cycles. However, this function is unknown.

The chloroviruses also encode functional chitinases and chitosanases [62]. The discovery that members of the Chlorella genus have chitin in their walls $[63,64]$ was surprising because chitin is normally absent in green algae. One hypothesis is that components of Chlorella chitin metabolism were acquired horizontally from the chloroviruses [11].

PBCV-1 also encodes at least five putative glycosyltransferases, which probably participate in glycosylating the virus MCP $[51,65]$. In contrast to all other viruses that use the host machinery located in the endoplasmic reticulum (ER) and Golgi to glycosylate their glycoproteins, chloroviruses encode most, if not all, of the components required to glycosylate their MCPs. Furthermore, all ex- perimental results indicate that glycosylation occurs independent of the ER and Golgi $[51,65]$. For example, unlike viruses that acquire their glycoprotein(s) by budding through a plasma membrane (which become infectious only during their release from the host), intact infectious PBCV-1 particles accumulate inside its host $30-40 \mathrm{~min}$ before virus release [66]. The identification of the glycanlinked Asn residues in the PBCV-1 MCP provided additional evidence that MCP glycosylation does not involve host ER glycosyltransferases [15].Noneofthe MCP glycan-linked Asn residues reside in a NX(T/S) sequence commonly recognized by ER-and Golgi-located glycosyltransferases [67]. Finally, the MCP, like the PBCV-1 glycosyltransferases, lacks an ER or Golgi signal peptide. We hypothesize that PBCV-1 encodes some, if not all, of the machinery involved in glycosylating its $\mathrm{MCP}$ and that the process occurs independent of the ER and Golgi.

\section{Evolutionary history}

Phycodnaviruses are believed to have an ancient, common evolutionary ancestry with some other large dsDNA viruses such as the poxviruses (e.g. smallpox virus), asfarviruses, iridoviruses, ascoviruses and mimiviruses [68-70]. Collectively these viruses are referred to as nucleocytoplasmic large dsDNA viruses (NCLDVs). Comparative analysis of 45 NCLDVs identified five genes common to all the viruses and 177 additional genes exist in at least two of the virus families [71].

Although a common ancestry of NCLDVs is generally accepted, there is lively discussion on the role these viruses played in the evolution of eukaryotes. These discussions, which are likely to continue for some time, include: (i) should NCLDVs be included in the tree of life? See [72- 75], comments by seven groups in Nat. Rev. Microbiol. (2009) 7, 614-627. (ii) Did the NCLDV genes arise 
from the original gene pool that led to prokaryotes and eukaryotes [69]? (iii) Did primitive NCLDVs give rise to the eukaryotic nucleus or vice versa, e.g. $[76,77]$ ? (iv) Does the structure of chlorovirus $\mathrm{MCP}$, which resembles MCPs from smaller dsDNA viruses with hosts in all three domains of life (human adenovirus, bacteriophage PRD1, and Archaea virus STIV), indicate these three viruses have a common evolutionary ancestor with the NCLDVs, despite the lack of amino acid sequence similarity among their MCPs, e.g. [78,79]?

\section{Concluding remarks}

With the increasing interest in using algae for producing biofuels, it is obvious that pathogens, including viruses, will affect yields [80]. Although this review focuses on certain large dsDNA viruses that infect algae, ssRNA, dsRNA and ssDNA viruses have recently been discovered that infect algae, e.g. [2]. Algal viruses are potentially a greater problem than plant viruses are to higher plants because plant viruses are usually transmitted by biological vectors. Thus two components are required for a virus disease outbreak in plants, whereas there is no evidence of biological vectoring of algal viruses; thus they only require one component for infection.

Chloroviruses are also a largely unexplored source of genetic elements for engineering algae and higher plants. For example, promoter elements from chloroviruses function well in both monocots and dicots of higher plants, as well as bacteria [81]. Also a translational enhancer element from a chlorella virus functions well in Arabidopsis [82].

The hosts for some algal viruses have been recently sequenced or are in the process of being sequenced. Annotation of these sequences will certainly contribute to studies on these viruses. However, one obstacle to studying the phycodnaviruses is that currently their hosts cannot be transformed. The development of successful and reproducible host transformation procedures will allow molecular genetic analysis of these viruses, which will be a major development.

Acknowledgments - We thank Dr. Leslie Lane for critical reading of the manuscript, and Dr. Alexander Tchourbanov for composing the gene map, and Wim van Egmond for the micrograph of zoochlorella-paramecium. Research in the Van Etten laboratory was supported in part by NSF-EPSCoR EPS1004094, DOE DE-FG36-08GO88055, DOE DE-EE0003142 and NIH/NCRR 1P20RR15635 from the COBRE program of the National Center for Research Resources.

\section{References}

1 Hull, R. (2009). Comparative Plant Virology, Elsevier/Academic Press.

2 Nagasaki, K. (2008). Dinoflagellates, diatoms, and their viruses. J. Microbiol. 46: 235-243.

3 Fuhrman, J.A. (1999). Marine viruses and their biogeochemical and ecological effects. Nature 399: 541-548.

4 Suttle, C.A. (2005). Marine viruses-major players in the global ecosystem. Nat. Rev. Microbiol. 5: 801-812.

5 Wommack, K.E. and Colwell, R.R. (2000). Viroplankton: viruses in aquatic ecosystems. Microbiol. Mol. Biol. Rev. 64: 69-114.

6 Dunigan, D.D. et al. (2006). Phycodnaviruses: a peek at genetic diversity. Virus Res. 117: 119-132.

7 Van Etten, J.L. (2003). Unusual life style of giant chloroviruses. Annu. Rev. Genet. 37: 153-195.
8 Wilson, W.H. et al. (2009). The Phycodnaviridae: the story of how tiny giants rule the world. In: Lesser Known Large dsDNA Viruses, J. Van Etten, editor, pp. 1-42, Springer.

9 Yamada, T. et al. (2006). Chloroviruses. In: Advances in Virus Research (Vol. 66), K. Maramorosch and A.J. Shatkin, editors, pp. 293-366, Elsevier.

10 Proschold, T. et al. (2011). The systematics of zoochlorella revisited employing an integrative approach. Environ. Microbiol. 13: 350-364.

11 Blanc, G. et al. (2010). The Chlorella variabilis NC64A genome reveals adaptation to photosymbiosis, coevolution with viruses, and cryptic sex. Plant Cell 22: 2,943-2,955.

12 Yan, X. et al. (2000) Structure and assembly of large lipid-containing dsDNA viruses. Nat. Struct. Biol. 7, 101-103.

13 Cherrier, M.V. et al. (2009). An icosahedral algal virus has a complex unique vertex decorated by a spike. Proc. Natl. Acad. Sci. U.S.A. 106: 11,085-11,089.

14 Zhang, X. et al. (2011). Three-dimensional structure and function of the Paramecium bursaria chlorella virus capsid. Proc. Natl. Acad. Sci. U.S.A. 108: 14,837-14,842.

15 Nandhagopal, N. et al. (2002). The structure and evolution of the major capsid protein of a large, lipid-containing, DNA virus. Proc. Natl. Acad. Sci. U.S.A. 99: 14,758-14,763.

16 Thiel, G. et al. (2010). Initial events associated with virus PBCV-1 infection of Chlorella NC64A. In: Progress in Botany (Vol. 71), U. Lüttge, et al., editors, pp. 169-183, Springer-Verlag.

17 Agarkova, I.V. et al. (2008). Chlorovirus-mediated membrane depolarization of chlorella alters secondary active transport of solutes. J. Virol. 82: 12,181-12,190.

18 Greiner, T. et al. (2009). Chlorella viruses prevent multiple infections by depolarizing the host membrane. J. Gen. Virol. 90: $2,033-2,039$.

19 Agarkova, I.V. et al. (2006). Virion-associated restriction endonucleases of chloroviruses. J. Virol. 80: 8,114-8,123.

$20 \mathrm{Mujtaba}$, S. et al. (2008). Epigenetic transcription repression of cellular genes by a viral SET protein. Nat. Cell Biol. 10: 1,114-1,122.

21 Iyer, L.M. et al. (2004). Comparative genomics of the FtsK-HerA superfamily of pumping ATPases: implications for the origins of chromosome segregation, cell division and viral capsid packaging. Nucleic Acids Res. 32: 5,260-5,279.

22 Bidle, K.D. et al. (2007). Viral activation and recruitment of metacaspases in the unicellular coccolithophore, Emiliania huxleyi. Proc. Natl. Acad. Sci. U.S.A. 104: 6,049-6,054.

23 Bidle, K.D. and Vardi, A. (2011). A chemical arms race at sea mediates algal host-virus interactions. Curr. Opin. Microbiol. 14: 449-457.

24 Yanai-Balser, G.M. et al. (2010). Microarray analysis of Paramecium bursaria chlorella virus 1 transcription. J. Virol. 84: 532-542.

25 Landstein, D. et al. (1995). Large deletions in antigenic variants of the chlorella virus PBCV-1. Virology 214: 413-420.

26 Van Etten, J.L. et al. (1991). Viruses and virus-like particles of eukaryotic algae. Microbiol. Rev. 55: 586-620.

27 Nelson, M. et al. (1998). Chlorella viruses encode multiple DNA methyltransferases. Biol. Chem. 379: 423-428.

28 Chan, S.H. et al. (2006). Cloning of Nt.CviQII nicking endonucleases and its cognate methyltransferase: M. CviQII methylates AG sequences. Prot. Expr. Purif. 49: 138-150.

29 Fitzgerald, L.A. et al. (2007). Sequence and annotation of the 369-kb NY-2A and 345-kb AR158 viruses that infect Chlorella NC64A. Virology 358: 472-484.

30 Fitzgerald, L.A. et al. (2007). Sequence and annotation of the 314-kb MT325 and the 321-kb FR483 viruses that infect Chlorella Pbi. Virology 358: 459-471.

31 Fitzgerald, L.A. et al. (2007). Sequence and annotation of the 288-kb ATCV-1 virus that infects an endosymbiotic chlorella strain of the heliozoon Acanthocystis turfacea. Virology 362: 350-361.

32 Fitzgerald, L.A. et al. (2008). Putative gene promoter sequences in the chloroviruses. Virology 380: 388-393.

33 Ho, C.K. et al. (1997). Characterization of an ATP dependent DNA ligase encoded by chlorella virus PBCV-1. J. Virol. 71: 1,931-1,937.

34 Lavrukhin, O.V. et al. (2000). Topoisomerase II from chlorella virus PBCV-1. Characterization of the smallest known type II topoisomerase. J. Biol. Chem. 275: 6,915-6,921. 
35 Eriksson, M. et al. (1999). Evidence for 4-hydroxproline in viral proteins: characterization of a viral prolyl 4 hydroxylase and its peptide substrates. J. Biol. Chem. 274: 22,131-22,134.

36 Manzur, K.L. et al. (2003). A dimeric viral SET domain methyltransferase specific to Lys27 of histone H3. Nat. Struct. Biol. 10: 187-196.

37 Plugge, B. et al. (2000). A potassium channel protein encoded by chlorella virus PBCV-1. Science 287: 1,641-1,644.

38 Gazzarrini, S. et al. (2009). Eighty-two amino acids are sufficient for making a potassium selective, voltage-dependent channel. Biochem. J. 420: 295-303.

39 Pagliuca, C. et al. (2007). Molecular properties of Kcv, a virus encoded K+ channel. Biochemistry 46: 1079-1090.

40 Shim, J.W. et al. (2007). In vitro synthesis, tetramerization and single channel characterization of virus-encoded potassium channel Kcv. FEBS Lett. 581: 1,027-1,034.

41 Chatelain, F.C. et al. (2009). Selection of inhibitor-resistant viral potassium channels identifies a selectivity filter site that affects barium and amantadine block. PLOS ONE 4: e7496.

42 Hertel, B. et al. (2010). Salt bridges in the miniature viral channel Kcv are important for function. Eur. Biophys. J. 39: 1,057-1,068.

43 Balss, J. et al. (2008). Transmembrane domain length of viral $\mathrm{K}^{+}$ channels is a signal for mitochondria targeting. Proc. Natl. Acad. Sci. U.S.A. 105: 12,313-12,318.

44 Thiel, G. et al. (2011). Minimal art: or why small viral $\mathrm{K}^{+}$channels are good tools for understanding basic structure and function relations. Biochem. Biophys. Acta 1808: 580-588.

45 Gazzarrini, S. et al. (2006). Chlorella virus MT325 encodes water and potassium channels that interact synergistically. Proc. Natl. Acad. Sci. U.S.A. 103: 5,355-5,360.

46 Bonza, M.C. et al. (2010). Functional calcium transporting ATPase encoded by chlorella viruses. J. Gen. Virol. 91: 2620-2629.

47 Greiner, T. et al. (2011). A functional HAK/KUP/KT-like potassium transporter encoded by chlorella viruses. Plant J. 68: 977-986.

48 Kaiser, A. et al. (1999). Chlorella virus PBCV-1 encodes a functional homospermidine synthase. Virology 263: 254-262.

49 Shah, R. et al. (2004). Chlorella virus PBCV-1 encodes an unusual arginine decarboxylase that is a close homolog of eukaryotic ornithine decarboxylases. J. Biol. Chem. 279: 35,760-35,767.

50 Baumann, S. et al. (2007). Chlorella viruses contain genes encoding a complete polyamine biosynthesis pathway. Virology 360: 209-217.

51 Van Etten, J.L. et al. (2010). Chloroviruses encode most, if not all, of the machinery to glycosylate their glycoproteins independent of the endoplasmic reticulum and Golgi. Biochim. Biophys. Acta 1800: 152-159.

52 Fruscione, F. et al. (2008). Differential role of NADP ${ }^{+}$and NADPH in the activity and structure of GDP-D-mannose 4,6-dehydratase from two chlorella viruses. J. Biol. Chem. 283: 184-193.

53 Tonetti, M. et al. (2003). Chlorella virus PBCV-1 encodes two enzymes involved in the biosynthesis of GDP-L-fucose and GDP-Drhamnose. J. Biol. Chem. 278: 21,672-21,677.

54 Parakkottil, C.M. et al. (2010). Identification of an L-rhamnose synthetic pathway in two nucleocytoplasmic large DNA viruses. J. Virol. 84: 8,829-8,838.

55 DeAngelis, P.L. et al. (1997). Hyaluronan synthase of chlorella virus PBCV-1. Science 278: 1800-1803.

56 Graves, M.V. et al. (1999). Hyaluronan synthesis in virus PBCV-1 infected chlorella-like green algae. Virology 257: 15-23.

57 DeAngelis, P.L. (1999). Hyaluronan synthases: fascinating glycosyltransferases from vertebrates, bacterial pathogens, and algal viruses. Cell Mol. Life Sci. 56: 670-682.

58 DeAngelis, P.L. (2002). Evolution of glycosaminoglycans and their glycosyltransferases: implication for the extracellular matrices of animals and the capsules of pathogenic bacteria. Anat. Rec. 268: 317-326.
59 Landstein, D. et al. (1998). Chlorella virus PBCV-1 encodes functional glutamine: fructose-6-phosphate amidotransferase and UDP-glucose dehydrogenase enzymes. Virology 250: 388-396.

60 Kawasaki, T. et al. (2002). Chitin synthesis in chlorovirus CVK2-infected chlorella cells. Virology 302: 123-131.

61 Yamada, T. and Kawasaki, T. (2005). Microbial synthesis of hyaluronan and chitin: new approaches. J. Biosci. Bioeng. 99: 521-528.

62 Sun, L. et al. (1999). Characterization of two chitinase genes and one chitosanase gene encoded by chlorella virus PBCV-1. Virology 263: 376-387.

63 Takeda, H. (1991). Sugar composition of the cell wall and the taxonomy of Chlorella (Chlorophyceae). J. Phycol. 27: 224-232.

64 Kapaun, E. et al. (1992). Cell wall composition of virus-sensitive symbiotic Chlorella species. Phytochemistry 31, 3,103-3,104.

65 Wang, I.N. et al. (1993). Evidence for virus-encoded glycosylation specificity. Proc. Natl. Acad. Sci. U.S.A. 90: 3,840-3,844.

66 Van Etten, J.L. et al. (1983). Growth cycle of a virus, PBCV-1, that infects chlorella-like algae. Virology 126: 117-125.

67 Reuter, G. and Gabius, H.J. (1999). Eukaryotic glycosylation: whim of nature or multipurpose tool. Cell Mol. Life Sci. 55: 368-422.

68 Iyer, L.M. et al. (2001). Common origin of four diverse families of large eukaryotic DNA viruses. J. Virol. 75: 11,720-11,734.

69 Iyer, L.M. et al. (2006). Evolutionary genomics of nucleo-cytoplasmic large DNA viruses. Virus Res. 117: 156-184.

70 Koonin, E.V. and Yutin, N. (2010). Origin and evolution of eukaryotic large nucleo-cytoplasmic DNA viruses. Intervirology 53: 284-292.

71 Yutin, N. et al. (2009). Eukaryotic large nucleo-cytoplasmic DNA viruses: clusters of orthologous genes and reconstruction of viral genome evolution. Virol. J. 6: 223.

72 Moreira, D. and López-García, P. (2009). Ten reasons to exclude viruses from the tree of life. Nature Rev. Microbiol. 7: 306-311.

73 Boyer, M. et al. (2010). Phylogenetic and phyletic studies of informational genes in genomes highlight existence of a $4^{\text {th }}$ domain of life including giant viruses. PLOS ONE 5: e15530.

74 Villarreal, L.P. and Witzany, G. (2010). Viruses are essential agents within the roots and stem of the tree of life. J. Theor. Biol. 262: 698-700.

75 Van Etten, J.L. (2011). Giant viruses. Am. Sci. 99: 304-311.

76 Bell, P.J.L. (2001). Viral eukaryogenesis: was the ancestor of the nucleus a complex DNA virus? J. Mol. Evol. 53: 251-256.

77 Takemura, M. (2001). Poxviruses and the origin of the eukaryotic nucleus. J. Mol. Evol. 52: 419-425.

78 Krupovic, M. and Bamford, D. (2008). Virus evolution: how far does the double b-barrel viral lineage extend? Nat. Rev. Microbiol. 6: 941-948.

79 Holmes, E.C. (2011). What does virus evolution tell us about virus origins? J. Virol. 85: 5,247-5,251.

80 Gachon, C.M.M. et al. (2010). Algal diseases: spotlight on a black box. Trends Plant Sci. 15: 633-640.

81 Mitra, A. et al. (1994). A chlorella virus gene promoter functions as a strong promoter both in plants and bacteria. Biochem. Biophys. Res. Commun. 204: 187-194.

82 Nguyen, P.S. et al. (2009). The A312L 5'-UTR of chlorella virus PBCV-1 is a translational enhancer in Arabidopsis thaliana. Virus Res. 140: 138-146.

83 Meints, R.H. et al. (1984). Infection of a Chlorella-like alga with the virus, PBCV-1: ultrastructure studies. Virology 138: 341-346.

84 Meints, R.H. et al. (1986). Assembly site of the virus PBCV-1 in a Chlorella-like green alga: ultrastructural studies. Virology 154: 240-245.

85 Stothard, P. and Wishart, D.S. (2005). Circular genome visualization and exploration using CGView. Bioinformatics 21: 537-539. 